\title{
Numerical simulation of transient electro-quasistatic fields using advanced subspace projection techniques
}

\author{
T. Steinmetz, G. Wimmer, and M. Clemens
}

Chair for Theory of Electrical Engineering and Computational Electromagnetics, Helmut-Schmidt-University, University of the Federal Armed Forces Hamburg, P.O. Box 700822 D-22008 Hamburg, Germany

\begin{abstract}
The transient simulation of electro-quasistatic fields requires a formulation that takes both dielectric effects as well as nonlinear conductive effects into account. The successive solution of large linear systems of equations with similar or even identical system matrices has to be performed repeatedly if this formulation is discretized by the FiniteElement method and an Implicit-Runge-Kutta method, respectively. The solution processes can be accelerated by using subspace recycling techniques and subspace projection extrapolation techniques. Numerical results for threedimensional high-voltage applications are presented and the efficiency of these techniques is shown.
\end{abstract}

\section{Introduction}

For the analysis of technical devices operated by using slowly varying electromagnetic fields, electromagnetic wave propagation can be neglected if the characteristic dimension of the device under consideration is much smaller than the distance an electromagnetic wave covers during the characteristic time of the device. The characteristic time is the reciprocal of the highest frequency at which the device is operated (Dirks, 1996). This leads to the wellknown quasistationary approximation to Maxwell's equations where changes in the current or charge distribution have effect instantly in space. This can be enforced in Maxwell's equations by discarding the solenoidal part of the displacement current in Ampere's law while keeping its irrotational part. Whereas in the static case, i.e. all time derivatives occuring in Maxwell's equations vanish $\left(\frac{\partial}{\partial t} \boldsymbol{D} \equiv \frac{\partial}{\partial t} \boldsymbol{B} \equiv 0\right)$, the quasistationary approximation can further be classified in electro-quasistatic fields (EQS) and magneto-quasistatic fields (MQS) considering the governing electric or magnetic

Correspondence to: T. Steinmetz

(thorsten.steinmetz@hsu-hh.de) energy density $w_{\mathrm{e}}$ and $w_{\mathrm{m}}$, respectively. If the electric energy density $w_{\mathrm{e}}$ exceeds the magnetic energy $w_{\mathrm{m}}$ for a given problem, the electro-quasistatic assumption is applicable, whereas the magneto-quasistatic assumption is applicable for $w_{\mathrm{m}} \gg w_{\mathrm{e}}$. The EQS assumption is expressed in Maxwell's equations by discarding the induction voltage in Faraday's law, $\frac{\partial}{\partial t} \boldsymbol{B} \equiv 0$. Typically, high electric energy densities and low magnetic energy densities occur if the dielectric relaxation time $\tau_{\text {relax }}=\varepsilon / \kappa$ is much greater and the magnetic diffusion time $\tau_{\text {diff }}=\mu \kappa d^{2}$ is much smaller than the device's characteristic time. Here, the permeability $\mu$, the permittivity $\varepsilon$, the conductivity $\kappa$ and the characteristic spatial dimension $d$ are used.

\section{Transient electro-quasistatic fields}

Faraday's law under the EQS assumption yields

$\operatorname{rot} \boldsymbol{E}=0$.

Hence, a description of electro-quasistatic fields is possible by a scalar potential function $\varphi(\boldsymbol{r})$ with $\boldsymbol{E}(\boldsymbol{r})=-\operatorname{grad} \varphi(\boldsymbol{r})$. Looking at Ampere's law,

$\operatorname{rot} \boldsymbol{H}=\boldsymbol{J}+\frac{\partial}{\partial t} \boldsymbol{D}$,

together with the material constitutive laws,

$\boldsymbol{D}(\boldsymbol{r}, t)=\varepsilon \boldsymbol{E}(\boldsymbol{r}, t)$,

$\boldsymbol{J}(\boldsymbol{r}, t)=\kappa(\boldsymbol{E}(\boldsymbol{r}, t)) \boldsymbol{E}(\boldsymbol{r}, t)$,

a conditional equation for the scalar potential $\varphi$ under the EQS assumption follows,

$\operatorname{div}(\kappa(\varphi) \operatorname{grad} \varphi)+\operatorname{div}\left(\varepsilon \operatorname{grad} \frac{\partial}{\partial t} \varphi(t)\right)=0$.

This equation is geometrically discretized using the FiniteElement method, which results in a nonlinear system of stiff 
ordinary differential equations in the time domain, which can be represented by the matrix equation

$\mathbf{K}_{\kappa}(\Phi) \Phi+\mathbf{B}_{\varepsilon} \frac{\mathrm{d}}{\mathrm{d} t} \Phi=0$,

where the matrices $\mathbf{K}$ and $\mathbf{B}$ are finite-element stiffness matrices of the electrical conductivity and the electric permittivity, respectively. Moreover, $\Phi$ denotes the vector of the discrete scalar potential and $\Phi\left(t_{0}\right)=\Phi_{0}$ its initial value at time $t_{0}$.

\section{Adaptive time integration}

\subsection{Runge-Kutta methods}

In order to solve the resulting initial boundary value problem, time discretization based on Singly-Diagonal-ImplicitRunge-Kutta Methods (SDIRK) is performed (Clemens et al., 2004a; Lang, 2001). SDIRK methods are stiffly-accurate one-step methods with $s$ internal stages which discretize equation (1) to systems of equations of the type

$$
\begin{aligned}
\left(\mathbf{K}_{\kappa}\left(\Phi_{i}^{(n)}\right)+\frac{1}{a_{i i} \Delta t_{n}} \mathbf{B}_{\varepsilon}\right) \Phi_{i}^{(n)} & \\
& =\frac{1}{a_{i i} \Delta t_{n}} \mathbf{B}_{\varepsilon}\left(\Phi^{(n)}+\sum_{j=1}^{i-1} a_{i j} \Phi_{j}^{\prime(n)}\right),
\end{aligned}
$$

with stage derivative values

${\Phi^{\prime}}_{i}^{(n)}=\frac{\left(\Phi_{i}^{(n)}-\Phi^{(n)}-\sum_{j=1}^{i-1} a_{i j} \Phi_{j}^{\prime(n)}\right)}{a_{i i}}$

and $\Phi_{s}^{(n)}=\Phi^{(n+1)}$. The time step length is denoted by $\Delta t$ while $a_{i j}$ denotes coefficients of the specific SDIRK method. In addition to the solution vector $\Phi^{(n+1)}$ at the time instant $t_{n+1}$, embedded SDIRK methods provide a second solution vector $\bar{\Phi}^{(n+1)}$. Both solution vectors differ since they belong to different orders of convergence $p$ for $\Phi^{(n+1)}$ and $\bar{p}$ for $\bar{\Phi}^{(n+1)}$, respectively. Here, an SDIRK method with four internal stages and the orders of convergence $p=3$ and $\bar{p}=2$ is used.

\subsection{Adaptive time step selection}

The local truncation error of a time step can be estimated by

$\left\|\Phi^{(n+1)}\right\|_{\mathrm{err}}=\sqrt{\frac{\left\|\Phi^{(n+1)}-\mathbf{\Phi}^{(n+1)}\right\|_{\infty}^{2}}{\left\|\Phi^{(n+1)}\right\|_{\infty}^{2}+\delta}}$,

with $\delta$ as an absolute error tolerance computed by $\delta=\theta \cdot \max _{t \leq t^{(n)}}\left(\|\Phi(t)\|_{\infty}^{2}\right), \theta \in\left[10^{-3}, 10^{-2}\right]$. This error estimate can be used twice: First, one can decide whether the time step leads to an acceptable local truncation error. Then the computed time step solution is accepted. If the local truncation error is greater than a given tolerance, the time step is

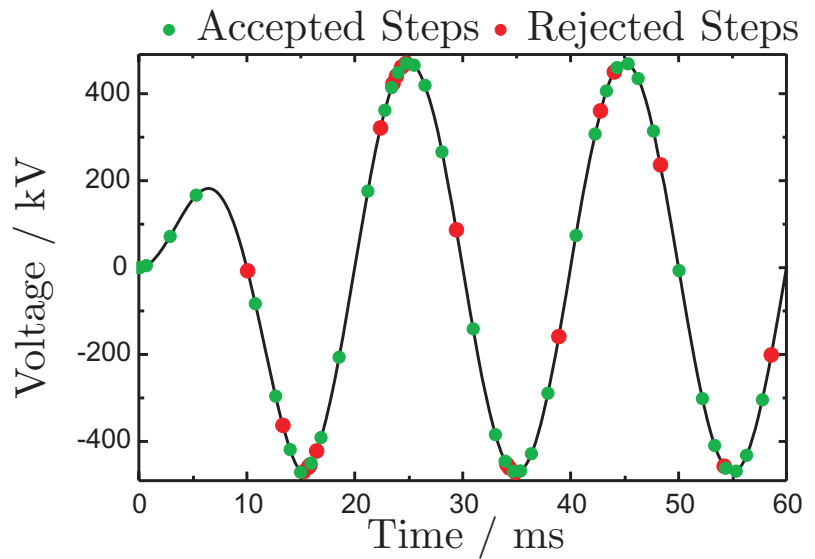

Fig. 1. Transient adaptive EQS simulation, displayed are accepted and rejected time steps.

rejected, i.e. a new time step is performed starting again from the previous time $t_{n}$. In both cases, the error estimate can be used to predict the next time step length

$\Delta t_{n+1}=v\left(\frac{r t o l}{\left\|\Phi^{(n+1)}\right\|_{\mathrm{err}}}\right)^{\frac{1}{p}} \Delta t_{n}$,

using a closed loop model of an I-controller scheme (Gustafsson, 1994; Clemens et al., 2002) with rtol as the required relative tolerance. Fig. 1 shows a transient simulation with adaptive choice of the times step length for a ramped sinusoidal excitation signal.

\subsection{Solution of the algebraic systems of equations}

In each stage of the time integration scheme a (non)linear algebraic system of equations of the form

$$
\left(\mathbf{K}\left(\Phi_{i}^{(n)}\right)+\frac{1}{a_{i i} \Delta t^{(n)}} \mathbf{B}\right) \Phi_{i}^{(n)}=\mathbf{f}\left(\Phi^{(n)}, \ldots\right)
$$

has to be solved. After a linearization by a Newton-Raphson method, the solution of a sequence of linear algebraic systems of equations $\mathbf{A}^{l} \mathbf{x}^{l}=\mathbf{b}^{l}$ with $\mathbf{A}^{l}=\mathbf{A}\left(\mathbf{x}^{l-1}\right), l=1,2, \ldots$, and a start vector $\mathbf{x}_{0}$ is performed. In the following, the superscript $l$ is omitted. As the system matrices $\mathbf{A}^{l}$ are symmetric, positive definite and sparse, a preconditioned conjugategradient method (PCG) is a suitable iterative solver for this type. Thereby, the PCG method generates iteration vectors

$\mathbf{x}_{i} \in \mathbf{x}_{0}+K_{i}\left\{\mathbf{M}^{-1} \mathbf{A}, \mathbf{M}^{-1} \mathbf{r}_{0}\right\}$.

with $K_{i}\left\{\mathbf{M}^{-1} \mathbf{A}, \mathbf{M}^{-1} \mathbf{r}_{0}\right\}$ denoting the $i$-th Krylov space which is constructed iteratively within the PCG method. The preconditioning operator is denoted by $\mathbf{M}$ which is a spectral approximation of the system matrix A. Here an algebraic multigrid method (AMG) is used as preconditioner. 
The speed of convergence of the PCG method depends on the condition number $\sigma(\mathbf{A})$ of the system matrix $\mathbf{A}$ at the one hand and on the choice of the start vector $\mathbf{x}_{0}$ at the other hand,

$\left\|\mathbf{x}-\mathbf{x}_{i}\right\|_{\mathbf{A}} \leq 2\left\|\mathbf{x}-\mathbf{x}_{0}\right\|_{\mathbf{A}}\left(\frac{\sqrt{\sigma}-1}{\sqrt{\sigma}+1}\right)^{\sigma}$,

with $\|w\|_{\mathbf{A}}=\sqrt{w^{\mathrm{T}} \mathbf{A} w}$ and $\sigma(\mathbf{A})$ as the ratio of the highest and the lowest eigenvalue of the matrix $\mathbf{A}, \sigma(\mathbf{A}):=\lambda_{n} / \lambda_{1}$ (Golub and van Loan, 1996).

\section{Subspace projection techniques}

\subsection{Subspace projection extrapolation}

As shown above, a sequence of linear systems of equations has to be solved during a simulation. The generic way to do this is to treat these linear systems without any correlation. However, especially in the case of linear material behavior, the systems are very well correlated. Hence, the information gained in the solution process of one linear system can be reused in the following solution processes. In order to recycle this information, the proposed subspace projection extrapolation (SPE) approach in Clemens et al. (2004b) is to calculate the optimal linear combination of former solution vectors which is used to generate a start vector for the current solution process.

In this paper we recycle the information of the Krylov space which is spanned by the preconditioned residual vectors $\mathbf{z}_{1}, \ldots, \mathbf{z}_{m}$ constructed by the PCG method while solving the first linear system of equations during a transient simulation. This Krylov space contains approximations of eigenvectors of the matrix $\mathbf{A}$ associated with the smallest eigenvalues of $\mathbf{A}$. The corresponding matrix operator $\mathbf{Q}$ is built by arranging the Krylov basis vectors as columns vectors of $\mathbf{Q}$,

$\mathbf{Q}=\left\{\mathbf{z}_{1}|\ldots| \mathbf{z}_{m}\right\} \in \mathbb{R}^{n \times m}$.

Here, the application of a good preconditioner is crucial, because it is very important that the dimension of the matrix operator $\mathbf{Q}$ is low, otherwise the numerical costs of the following projection techniques may outweigh their profits. The matrix $\mathbf{Q}$ can then be used to solve the Galerkin-projected linear system

$$
\mathbf{Q}^{T} \mathbf{A} \mathbf{Q z}=\mathbf{Q}^{T} \mathbf{b}
$$

instead of the originally system $\mathbf{A x}=\mathbf{b}$ in order to compute a start vector

$\mathbf{x}_{0}^{\mathrm{SPE}}:=\mathbf{Q z}=\mathbf{Q}\left(\mathbf{Q}^{T} \mathbf{A Q}\right)^{-1} \mathbf{Q}^{T} \mathbf{b}$

for the respective PCG iteration. Through this proceeding, the start vector $\mathbf{x}_{0}^{\mathrm{SPE}}$ already contains information associated with eigenvectors corresponding with the $k$ smallest eigenvalues $\lambda_{1}, \ldots, \lambda_{k}$ of the system matrix $\mathbf{A}$. The PCG method will therefore converge with the effective condition number $\sigma_{\text {eff }}$ instead of the condition number $\sigma(\mathbf{A})$ of the system matrix $\mathbf{A}$,

$\sigma_{\text {eff }}(\mathbf{A}):=\frac{\lambda_{n}}{\lambda_{k}}<\sigma(\mathbf{A}):=\frac{\lambda_{n}}{\lambda_{1}}$,

what can be understood as an implicit deflation of the PCG method.

\subsection{Augmented PCG method}

The solution processes can be accelerated additionally by not only generating a start vector reusing subspace information, but furthermore by modifying the PCG algorithm itself (Gosselet and Rey, 2002; Saad et al., 2000) in order to obtain residual vectors $\mathbf{r}_{i}$ which are orthogonal to the Krylov space spanned by the column vectors of $\mathbf{Q}$,

$\mathbf{Q}^{T} \mathbf{r}_{i}=0$.

This can be achieved by augmenting the $i$-th Krylov space by the range of $\mathbf{Q}$ resulting in iteration vectors

$\mathbf{x}_{i} \in \mathbf{x}_{0}+K_{i}\left\{\mathbf{M}^{-1} \mathbf{A}, \mathbf{M}^{-1} \mathbf{r}_{0}\right\} \oplus \operatorname{Range}\{\mathbf{Q}\}$.

Thus, using the augmented PCG method,

$\mathbf{r}_{i} \perp K_{i}\left\{\mathbf{M}^{-1} \mathbf{A}, \mathbf{M}^{-1} \mathbf{r}_{0}\right\} \oplus \operatorname{Range}\{\mathbf{Q}\}$

is valid. Defining the projector

$\mathbf{P}_{Q}=\mathbf{I}-\mathbf{Q}\left(\mathbf{Q}^{T} \mathbf{A} \mathbf{Q}\right)^{-1} \mathbf{Q}^{T} \mathbf{A}$

which projects vectors onto the A-orthogonal complement of the space spanned by the column vectors of $\mathbf{Q}, \mathbf{Q}^{T} \mathbf{r}_{i}=0$ is enforced if $\mathbf{P}_{Q}$ is applied to the preconditioned residual vectors in the Augmented PCG (AugPCG) iteration.

\subsection{Combined algorithm}

In Algorithm 1, the subspace projection extrapolation as well as the augmented PCG method are combined with atol $_{\mathrm{rhs}}=\max _{j=1, \ldots, l-1}\left\|\mathbf{b}^{j}\right\|$ as the maximum absolute value of the right-hand side vectors for all previous systems with $\Theta \in\left[10^{-3}, 10^{-2}\right]$ and an arbitrary start vector $\mathbf{x}_{00}$.

\section{Numerical results}

The first technical example shown in Fig. 2 is a high-voltage bushing which mainly consists of metal, ceramic insulators and insulation oil with a nonlinear electrical conducitvity characertistic $\kappa(\boldsymbol{E})$. The simulation with solely linear material behavior yields the result that the SPE-PCG with 13 column vectors in the matrix $\mathbf{Q}$ is more effective than the AugPCG method. This is due to the fact that the system matrices in the stages of the time integrator are nearly invariant.

As can be seen in Fig. 2, the plot of the scalar potential of the model with solely linear material behavior differs clearly 

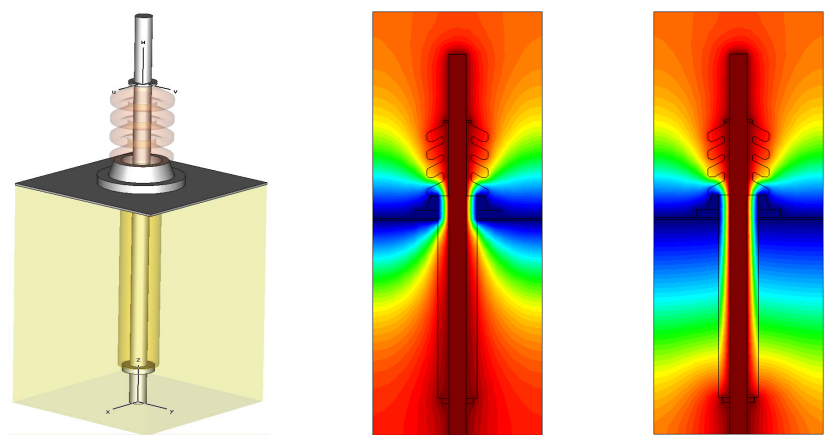

Fig. 2. HV Bushing: Geometry, plot of the scalar potential with linear and nonlinear electric conductivity (from left).

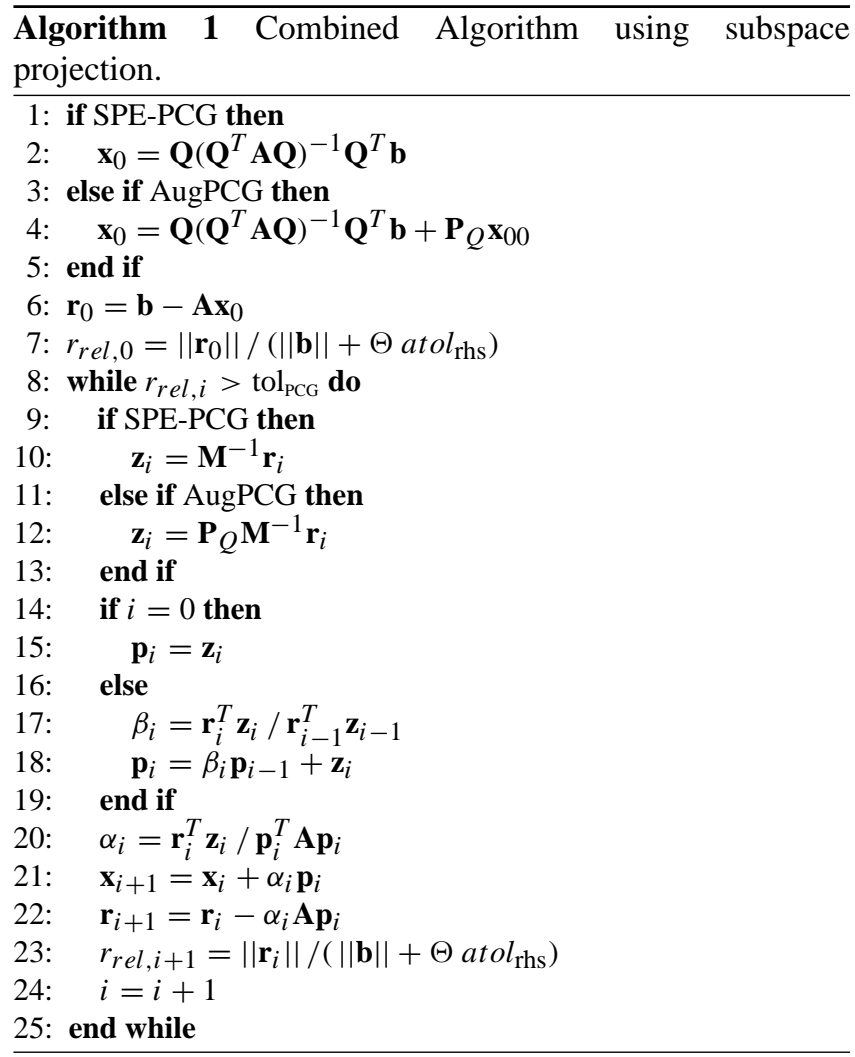

from the plot of the model with nonlinear electrical conductivity characteristic for the insulation oil. In that case, the AugPCG method, in which the projection matrix $\mathbf{P}_{Q}$ is updated by the system matrix $\mathbf{A}$ for each linear system, is more effective, as the system matrices differ from each other due to the nonlinearity. The number of column vectors in $\mathbf{Q}$ is 13 in a model with $53502 \mathrm{DoF}$ and 16 in a model with 162113 DoF. Unfortunately, although the number of PCG iterations is reduced obviously, the numerical costs of the projection leads to smaller benefits in the computation time needed to solve all linear systems in a transient simulation. The number of the PCG iterations and the related computation times
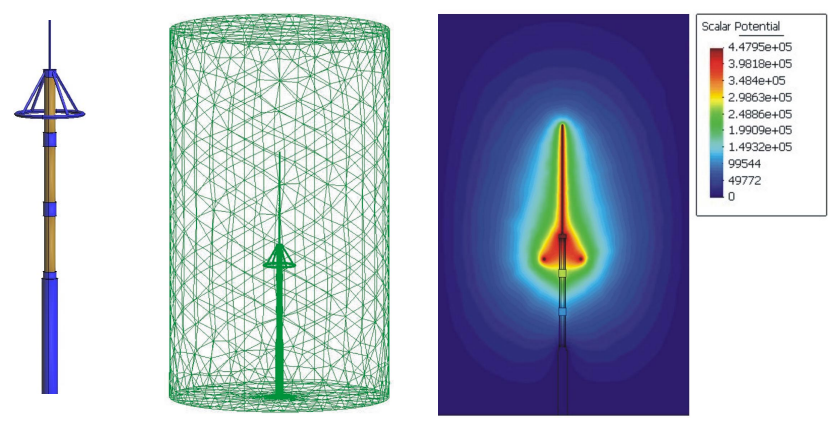

Fig. 3. HV surge arrester: Geometry, mesh and plot of the scalar potential (from left).

Table 1. Number of iterations and computation time for the HV bushing

\begin{tabular}{llll}
\hline Model & Alg. Var. & PCG-Iter. & Sol. time \\
\hline Linear & PCG & 2725 & $380.9 \mathrm{~s}$ \\
\cline { 2 - 4 } 53502 DoF & SPE-PCG & 49 & $19.6 \mathrm{~s}$ \\
\hline Non-Linear & PCG & 2748 & $391.4 \mathrm{~s}$ \\
\cline { 2 - 4 } 53502 DoF & AugPCG & 1199 & $336.5 \mathrm{~s}$ \\
\hline Non-Linear & PCG & 3344 & $1361.5 \mathrm{~s}$ \\
\cline { 2 - 4 } 162113 DoF & AugPCG & 1531 & $1214.7 \mathrm{~s}$
\end{tabular}

Table 2. Number of iterations and computation time for the HV surge arrester.

\begin{tabular}{llll}
\hline Model & Alg. Var. & PCG-Iter. & Sol. time \\
\hline Non-Linear & PCG & 4372 & $345.6 \mathrm{~s}$ \\
\cline { 2 - 4 } 41100 DoF & AugPCG & 1037 & $243.4 \mathrm{~s}$ \\
\hline Non-Linear & PCG & 7590 & $1846.7 \mathrm{~s}$ \\
\cline { 2 - 4 } 150590 DoF & AugPCG & 227 & $742.3 \mathrm{~s}$ \\
\hline
\end{tabular}

for the high-voltage bushing are shown in Table 1.

The second technical application is a high-voltage surge arrester shown in Fig. 3, a structure mainly consisting of metal, ceramic insulators and varistor material with a nonlinear electrical conductivity characteristic.

As in the first example, due to the nonlinearity the greatest acceleration in terms of CPU time is achieved by the AugPCG method in which the projection matrix with 21 column vectors for a model with $41100 \mathrm{DoF}$ and 44 column vectors for the model with $150590 \mathrm{DoF}$, respectively, is updated for each linear system. The number of the PCG iterations and the related computation times for the high-voltage surge arrester are shown in Table 2. 
The presented simulations are carried out on an double processor Intel Xeon $(2.8 \mathrm{GHz}, 2 \mathrm{~GB}$ RAM) with a constant time step length to gain a better comparability of the results.

\section{Conclusion}

A three-dimensional finite-element implementation based on a discrete formulation of electro-quasistatic fields was presented. Adaptive time integration based on an embedded Singly-Diagonal-Runge-Kutta method was performed. The repeated solution processes of the linear systems within the time integrator were accelerated using implicit and explicit subspace projection techniques. Their efficiency was shown in the transient simulation of technical applications.

\section{References}

Clemens, M., Wilke, M., and Weiland, T.: 3-D Transient Eddy Current Simulations Using FI $^{2}$ TD with Variable Time Step Size Selection Schemes, IEEE Trans. Magn., 38, 605-608, 2002.

Clemens, M., Wilke, M., Benderskaya, G., De Gersem, H., Koch, W., and Weiland, T.: Transient Electro-Quasi-Static Adap- tive Simulation Schemes, IEEE Trans. Magn., 240, 1294-1297, 2004a.

Clemens, M., Wilke, M., Schuhmann, R., and Weiland, T.: Subspace Projection Extrapolation Scheme for Transient Field Simulations, IEEE Trans. Magn., 40, 934-937, 2004 b.

Dirks, K. H.: Quasi-Stationary Fields for Microelectronic Applications, Electrical Engineering, 79, 145-155, 1996.

Golub, G. and van Loan, C.: Matrix Computations, Johns Hopkins University Press, London, 1996.

Gosselet, P. and Rey, C.: On a Selective Reuse of Krylov Subspaces in Newton-Krylov Approaches for Nonlinear Elasticity, in: Proc. of the 14th Intern. Conf. on Domain Decomposition Methods, edited by Herrera, I., Keyes, D. E., Widlund, O. B., and Yates, R., 419-426, 2002.

Gustafsson, K.: Control-Theoretic Techniques for Stepsize Selection in Implicit Runge-Kutta Methods, ACM Trans. Math. Software, 20, 496-517, 1994.

Lang, J.: Adaptive Multilevel Solution of Nonlinear Parabolic PDE Systems: Theory, Algorithm and Application, Springer, Berlin, Heidelberg, New York, 2001.

Saad, Y., Yeung, M., Erhel, J., and Guyomarch, F.: A Deflated Version of the Conjugate Gradient Algorithm, SIAM J. Sci. Comput., 21, 1909-1926, 2000. 